\title{
PRESENTACIÓN
}

\author{
INFANCIA, APRENDIZAJE Y FORMACIÓN
}

\author{
Julio César Arboleda ${ }^{1}$ \\ Grupo Redipe: Educación,
}

Los temas generales en los cuales se inscriben los artículos de este número de nuestra Revista Boletín Redipe son: infancia, aprendizaje y formación. El primer tema es tratado de manera particular en el artículo editorial que nos presenta la investigadora Sandra Lorena Arango. Se trata de una reflexión alrededor de una experiencia pedagógica bajo su liderazgo, basada en la investigación escolar, muy poco abordada en la escuela. Nos deja pensar en la necesidad inaplazable de avanzar propuestas pedagógicas para disminuir el pasivo subjetivo del niño en la educación y en el mundo invasor de los adultos. Es posible desarrollar estrategias de investigación en el aula en virtud de las cuales estas se conviertan en comunidades de indagación e investigación, que den lugar a experiencias ético estéticas que, al tiempo que permitan la construcción de conocimiento,

$1 \quad$ https://orcid.org/0000-0002-1572-5384 Estudios de pregrado en Literatura, Ciencias Sociales/ Historia; especialización en Docencia universitaria, magister y Doctor en Educación.

Director de Redipe: Red Iberoamericana de Pedagogía. Creador de la perspectiva Pedagogía de la comprensión edificadora. Grupo de Investigación REDIPE: Educación, Epistemología y Diversidad. direccion@redipe.org. https://scholar.google.com/citations?user=xeClbeYAAAAJ\& $\underline{h l=e s}$ potencien la infancia en los niños, eleven su subjetividad. Retos y desafíos de la educación para la infancia en un escenario donde cada vez el mundo de los niños es asaltado por el mundo del adulto, azotado a su vez por la lógica del consumo y la rentabilidad, abortando de este modo el derecho al desarrollo pleno de su personalidad.

Los siguientes artículos constituyen importantes aportaciones investigativas y crítico reflexivas en torno al segundo y tercer tema, además de factores que impactan los procesos de formación, enseñanza, aprendizaje, de la importancia de la formación y espacio de la opinión (Posada G., Pedro), y del uso del conocimiento para fortalecer las prácticas culturales (Rodríguez N., M).

EDITORIAL. LA EXPERIENCIA DE EXTRAÑAMIENTO EN LAS PROPUESTAS PARA EL DESARROLLO DE LA INFANCIA. Artículo Editorial a cargo de la investigadora Sandra Lorena Arango Cardona de la Fundación Casa Munay, Colombia. Socializa algunos apartados de una experiencia 
pedagógica en el nivel preescolar, en la que se tejió durante un año lectivo un formato verosímil en el cual los niños se instauraron en el rol de exploradores y abordaron la misión de rescatar a los personajes de los cuentos clásicos de los Hermanos Grimm que habían sido raptados por los Hombres Grises de la historia de Momo de Michael Ende. Esta propuesta tuvo como ejes el juego simbólico, propiciado porla intertextualidad, y la decisión de asumir el acto educativo desde una perspectiva poética. Comparte la noción de experiencia de extrañamiento desde el enfoque de Cortázar (literatura) y de Everaert-Desmedt (semiótica), que propicia la búsqueda, el análisis, la contrastación y el establecimiento de planes de acción, a la vez que convoca a los niños en el marco de relaciones cooperativas con pares y adultos, para alcanzar fines comunes y experimentar la emoción del hallazgo y la resolución colectiva del misterio.

\section{ALGUNOS FUNDAMENTOS SOBRE LA FORMACIÓN CONTINUA DEL PROFESORADO DESDE EL ENFOQUE} RADICAL E INCLUSIVO. Artículo de reflexión propositiva de Agustín de la Herrán Gascón, Universidad Autónoma de Madrid. Aborda algunos fundamentos de la formación continua del profesorado desde el "enfoque radical e inclusivo de la educación", constructo de su autoría. Intenta sistematizar algunas razones que puedan ser útiles para la investigación pedagógica. El enfoque radical e inclusivo de la formación o de la educación parte de que la Pedagogía y demás Ciencias de la Educación -así como los sistemas educativos y organismos internacionales de educación- desarrollan una perspectiva superficial, parcial e incompleta de la educación y de la formación. Esto ocurre por varias razones encadenadas, que también se trasladan a la formación continua del profesorado: se parte de un concepto equivocado, incompleto e insuficiente de educación; no se comprende en profundidad la formación humana, y se desatienden ámbitos formativos radicales que, como ocurre con las raíces de un árbol, permanecen fuera de la vista, pese a ser su parte más vital. El enfoque en cuestión, al ser inclusivo, integra lo que se hace -en este caso, la formación continua del profesorado consensuada técnico-reflexiva y crítica por la comunidad científica y profesional-, que se percibe como necesaria e insuficiente. En consecuencia, se añaden otras menos evidentes $\mathrm{y}$ otras radicales desde las que completar $\mathrm{y}$ comprender la formación del profesorado de una manera más cabal.

\section{DOCENTE VIRTUAL: FUNCIONES, CARACTERÍSTICAS $Y \quad$ PRÁCTICA} PEDAGÓGICA. Artículo de reflexión propositiva elaborado por la académicas ecuatorianas Carmen Siavil Varguillas Carmona y Angélica María Urquizo Alcívar, UNACH. Expone el resultado de una indagatoria sobre el referente teórico de las funciones, características y práctica pedagógica del docente en la virtualidad. Así mismo, presenta distinciones entre Tecnologías de la Información y la Comunicación, y Entornos Virtuales de Enseñanza y Aprendizaje (EVEA), como conceptuaciones que permiten contextualizar al docente en su desempeño virtual. Cabe destacar que la educación con modalidad a distancia mediada por las TIC con la aplicación de EVEA, requiere un cambio de rol del docente, pasando de guía de estudiantes a facilitador del uso de recursos y herramientas necesarias para explorar y elaborar nuevos conocimientos y destrezas; de esta manera, se convierte en gestor de recursos de aprendizaje, acentuando su papel de orientador. En esta vía, cumple funciones de líder mediador entre la nueva información y el aprendizaje del estudiante, lo que requiere que sea capaz de diseñar experiencias, propiciar situaciones que motiven el crecimiento cognoscitivo, planificar actividades que faciliten el aprendizaje autodirigido, la motivación, la contextualización de los contenidos en la vida real, el trabajo de tipo colaborativo y cooperativo, las interacciones 
sociales y las múltiples representaciones del contenido y la reflexión.

\section{ESFERA PÚBLICA Y OPINÓN PÚBLICA.}

Artículo de reflexión propositiva aportado por el Dr. Pedro Posada Gómez, Universidad Del Valle, Dpto De Filosofía. Manifiesta que la "opinión pública" habla mucho y da mucho de qué hablar. Sin embargo, el "sujeto" que aquí habla es difícil de precisar. ¿Qué o quién es la opinión pública? ¿Es un sujeto colectivo o muchos sujetos hablando detrás de una máscara común? ¿Cómo se construyó el concepto de opinión pública? ¿Quién puede hablar a nombre de la opinión pública? ¿Conducirla, engañarla, instrumentalizarla? Para abordar estas preguntas, seguiremos el estudio de $\mathrm{J}$. Habermas: Historia y crítica de la opinión pública (La transformación estructural de la vida pública) ${ }^{2}$. Bajo esta guía haré (1) una síntesis apretada de la reconstrucción habermasiana del proceso histórico en el cual se construye (se abre) el espacio público burgués, (2) abordaré el surgimiento de la prensa escrita y el paso de la opinión pública literaria a la opinión pública política, (3) en tercer lugar reseñaré el paso histórico del público lector al público consumidor de cultura, (4) por último, presento algunas reflexiones posteriores del autor, en su obra Teoría de la acción comunicativa (1982).

\section{PROYECTO DE INTERVENCIÓN COGNITIVA,} AFECTIVA Y SOCIAL, PARA LA MEJORA DE LOS PROCESOS FORMATIVOS DEL LICENCIADO EN EDUCACIÓN. Artículo de investigación elaborado por las académicas Gloria del Jesús Hernández Marín, Leticia Arias Gómez y Silvia Estela Yon Guzmán de

2 Jürgen Habermas (1962): Historia y crítica de la opinión pública (La transformación estructural de la vida pública) (HCOP). Editorial Gustavo Gili, Barcelona, 1981. Traducción de Antoni Domènech. Titulo original: Strukturwandel der Öffentlichkeit (Untersuchungen zu einer Kategorie der bürgerlichen Gesellschaft). Considero que sería preferible una traducción más fiel al original: La transformación estructural del espacio público (Investigación sobre una categoría de la sociedad burguesa). La expresión "Öffentlichkeit" que Domènech traduce como 'publicidad', será tratada en este escrito como "espacio público". (P. P.)
México. Refiere un diagnóstico que sustenta la elaboración de una propuesta de intervención orientada al uso de los principios y recursos para fortalecer el acompañamiento de los estudiantes en el Programa Institucional de Tutoría (PIT). Su diseño atiende las dimensiones: afectiva, cognitiva y social por medio de talleres dirigidos a estudiantes, en tutoría grupal multigrado, durante el Horario Institucional de Tutoría (HIT), desarrollada de manera colaborativa.

\section{PROCESO DE GLOBALIZACIÓN EN GERMEN: MODO DE PRODUCCIÓN} AMERICANO: RUTAS DE COMERCIO DEL ORO, EL MAÍZ, LA TURQUESA Y OTROS ARTEFACTOS CULTURALES. Artículo de reflexión propositiva elaborado por la académica antropóloga Mariángela Rodríguez Nicholls, investigadora de CIESAS D.F., México. Muestra la existencia de rutas de intercambio comercial, de informaciones, de personas y de artefactos culturales a lo largo de América, lo cual nos pone de presente que no existían culturas autárquicas o aisladas sino en una relación intensa en la que los pueblos en su migración de sur a norte viajaron siempre con sus "fondos de conocimiento" como los nombra Carlos Vélez Ibáñez. Los pueblos receptores no fueron pasivos ante el contacto con los "otros" sino que resignificaron diversas prácticas culturales en ese proceso cultural que implica una creación continua.

\section{EL PAPEL DE LA ACADEMIA EN LA TENSIÓN} ENTRE LAS VIEJAS $Y$ LAS NUEVAS FORMAS DE SER Y ESTAR FRENTE A LAS PANTALLAS. Artículo de reflexión propositiva de los docentes investigadores Leandro Arbey Giraldo Henao, Jaime Andrés Ballesteros y Luis Fernando Carrillo Holguín, Grupo de investigación ACDM, Universidad Tecnológica de Pereira-Colombia. Reflexiona sobre el papel de la academia en la tensión entre las viejas y las nuevas formas de ser y estar frente a las pantallas. Se fundamenta teóricamente en dos momentos cruciales que soportan el desarrollo 
investigativo, cuyos abordajes se conectan con las viejas y las nuevas formas de ser y estar frente a las pantallas. En lo primero, se integra con una perspectiva teórica e histórica de la televisión en el ámbito latinoamericano, y, en lo segundo, con postulados sobre las formas modernas en que los jóvenes estudiantes universitarios se relacionan con la pantalla televisiva, en particular, y las multipantallas, en lo global. Se inscribe en una investigación de tipo cualitativa, con apoyo cuantitativo desde un enfoque descriptivo-interpretativo, en función de una perspectiva pedagógica sobre el desconocimiento de una presencia efectiva de las nuevas formas de ser y estar frente a las pantallas, así como el nivel de implicación de los hábitos y las discursividades propias de los estudiantes objeto de estudio.

\section{EL TEXTO ARGUMENTATIVO BILINGÜE ESPAÑOL-INGLÉS $Y$ LAS ELECCIONES L I N G Ü Í S T I C O - D I S C U R S I V A S DIRECCIONADAS POR EL ESTILO DE APRENDIZAJE EN ESTUDIANTES EN FORMACIÓN BILINGÜE. Artículo de} investigación elaborado por la académica Neira Loaiza Villalba, Universidad del Quindío, Colombia. Presenta algunos resultados de un estudio doctoral, de enfoque cuantitativo y diseño no experimental y transversal, en el campo del bilingüismo escolar con relación al direccionamiento que los estilos de aprendizaje de estudiantes de licenciatura en lenguas modernas de nivel intermedio hacen con respecto a las elecciones lingüístico-discursivas por las que ellos optan en la escritura argumentativa en inglés y español. El estilo de aprendizaje predominante en la muestra de 47 estudiantes, a quienes se aplicó el cuestionario de estilos de aprendizaje de Kolb (1976), fue el divergente $(72.3 \%)$. Se evidenció, a través de un análisis de retórica contrastiva intraindividual (Liu y Furneaux, 2013), que en general el estilo de aprendizaje predominante en los estudiantes direccionó en gran medida algunas de sus elecciones lingüístico-discursivas en dos cartas petitorias en español e inglés. Sin embargo, se reconoce que no sólo el estilo de aprendizaje puede incidir en dichas escogencias sino que ellas también podrían ser influenciadas por la naturaleza de la lengua y por las características cognitivas y afectivas que la argumentación como habilidad psicolingüística de alto nivel lingüístico, cognitivo y metacognitivo- demanda.

\section{AFRONTAMIENTO DEL ESTRÉS $Y$ PROCRASTINACIÓN EN EL PERSONAL DOCENTE $Y$ ADMINISTRATIVO DE LA UNHEVAL - HUANUCO - 2012". Artículo} de investigación elaborado por la académica Artículo de investigación elaborado por los académicos Reynaldo Marcial, Ostos Miravaol y Abner Alfeo Fonseca Livias, Universidad Nacional Hermilio Valdizán, Perú, orientada a determinar la existencia de la procrastinación, que significa el aplazamiento del cumplimiento de la tarea solo por el gusto de dejarla para el final, en el personal docente y administrativo de la UNHEVAL y asimismo establecer la relación que presenta esta variable en función de los estilos de afrontamiento del estrés: enfocado en el problema, enfocado en la emoción y en función a otros estilos como son la negación, conductas inadecuadas y distracción.

\section{NIVEL DE ENGAGEMENT Y SU IMPLICANCIA} EN EL RENDIMIENTO ACADÉMICO EN ESTUDIANTES UNIVERSITARIOS DE CIENCIAS DE LA SALUD DE LA UNHEVALHUÁNUCO, 2015. Artículo de investigación a cargo de Miguel Alfredo Carrasco Muñoz y Cecilia Martínez Morales de UNHEVALHUÁNUCO, Perú. Refiere el proyecto dirigido a establecer la relación entre el nivel de engagement y el rendimiento académico que presentan los estudiantes de ciencias de la salud de la UNHEVAL y demostrar además la implicancia del nivel de engagement en el rendimiento académico de dichos estudiantes. Los resultados muestran en términos generales 
que no existe relación significativa entre el engagement y el rendimiento académico de los estudiantes universitarios por lo que se concluye que el engagement no incide en el rendimiento; sin embargo, haciendo un análisis por escuelas se ha encontrado que si existe una relación significativa entre el engagement y rendimiento académico en los estudiantes de Enfermería y Obstetricia respectivamente. 